\title{
Detour problem-solving behavior in rats with early lesions to the "general learning system"
}

\author{
ROBERT THOMPSON, DAVID HARMON, and JEN YU \\ Fairview State Hospital, Costa Mesa, California \\ and University of California Irvine Medical Center, Orange, California
}

\begin{abstract}
Recent studies involving visual and nonvisual discrimination habits suggest that the components of the rodent's general (nonspecific) learning system (a group of brain structures essential for normal acquisition of a wide range of laboratory tasks) include the regions of the globus pallidus, lateral thalamus, substantia nigra, midbrain central gray, median raphe, and pontine reticular formation. To determine whether these regions play a role in more complex kinds of learning, young rats previously subjected to selective lesions to these brain sites received five trials on each of three "climbing" detour problems. Performance on Trial 1 (a measure of response flexibility) as well as performance on Trials 2-5 (a measure of detour habit formation) of each problem was significantly impaired in those groups with damage to the globus pallidus, substantia nigra, median raphe, or pontine reticular formation. On the other hand, those groups with damage to the lateral thalamus or midbrain central gray failed to display consistent deficits in detour problemsolving behavior. This pattern of results suggests that the latter two structures can no longer be grouped with the globus pallidus, substantia nigra, median raphe, and pontine reticular formation as components of the rodent's general learning system.
\end{abstract}

The "general learning system" (an ensemble of neural structures conceived to play a significant role in the acquisition of a broad spectrum of laboratory tasks) of the rat brain has been proposed to comprise a number of subcortical regions, including those occupied by the globus pallidus, lateral thalamus, substantia nigra, midbrain central gray, median raphe, and pontine reticular formation (Thompson, Gallardo, \& Yu, 1983). This proposal is based on the findings that localized electrolytic lesions to any one of the foregoing regions in adult rats retard acquisition and reversal of both visual and nonvisual discrimination habits (Thompson, 1982a, 1982b; Thompson et al., 1983). The finding that weanling rats subjected to these lesions also show a nonspecific learning deficit (Thompson, Ramsay, \& Yu, 1984; Thompson \& Yu, 1983) suggests the possibility that the young brain-injured rat (one bearing lesions to the general learning system) may serve as an animal model for certain classes of mental retardation, at least to the extent that both the braininjured rat and the mental retardate evidence a nonspecific (generalized) learning impairment (Denny, 1964).

One of the questions arising from these data is whether the disruptive effects of selective lesions to the proposed components of the general learning system are restricted to "simple" associative learning, as gauged by conventional two-choice sensory discrimination tasks, or whether

The completion of this study was facilitated by a grant from the Rehabilitation Center for Brain Dysfunction, Irvine, California. The authors are affiliated with the Department of Physical Medicine and Rehabilitation of the University of California Irvine Medical Center. Requests for reprints should be sent to Robert Thompson, Fairview State Hospital, 2501 Harbor Boulevard, Costa Mesa, CA 92626. these effects extend to more complex kinds of learning. The finding that acquisition of a three-cul maze is impaired by discrete lesions to these components (Thompson et al., 1984; Thompson \& Yu, 1983) suggests the latter, but further information on this point would be desirable.

The present study was undertaken to determine whether lesions to the globus pallidus, lateral thalamus, substantia nigra, midbrain central gray, median raphe, or pontine reticular formation in weanling rats would impair performance on a series of "climbing" detour problems. According to the results of an earlier experiment (Thompson, Harmon, \& Yu, 1984a), performance on Trial 1 of these problems seems to be sensitive to a cognitive function that is qualitatively different from that underlying simple associative learning. This was shown by several groups of neocortically damaged rats whose performance on Trial 1 was inferior to that of the controls, but whose performance on Trials 2-5 (a measure of detour habit formation) closely approximated that of the controls. The nature of this cognitive function tapped by performance on Trial 1 is unclear, but it may be related to behavioral inhibition, exploration, recent (working) memory, or attention (see Thompson et al., 1984a).

As a control for any possible nonspecific debilitating effects arising from brainstem damage, a group of weanling rats with lesions to the lateral portions of the brainstem at pontomesencephalic levels was included in this study. (This region of the brainstem has not been found to be critical for acquisition of either visual or nonvisual discrimination habits-Thompson et al., 1984.) Since a defective labyrinthine righting reflex is often associated with medial brainstem damage (Thompson, Arabie, \& Sisk, 1976), a condition that may interfere with the ex- 
pression of a climbing response, a group of weanling rats with lesions to the medial longitudinal fasciculus at midbrain levels was also investigated. Finally, since the solution of our detour problems is conditional upon both exploratory activity and rearing responses, these behaviors were measured in an open-field test at the conclusion of the experiment.

\section{METHOD}

\section{Subjects and Surgery}

Weanling (22-24-day-old) male Sprague-Dawley albino rats, 40-65 g, underwent surgery under deep chloral hydrate anesthesia $(400 \mathrm{mg} / \mathrm{kg})$. All lesions were accomplished electrolytically (in one stage) by passing a constant anodal current of $1.0-1.8 \mathrm{~mA}$ for a duration of 8-10 sec through an implanted stainless steel electrode $(0.5 \mathrm{~mm}$ in diam) with $0.5-1.0 \mathrm{~mm}$ of the tip exposed. One group sustained a midline lesion to the median raphe (Group MR), and seven groups received bilateral lesions to the globus pallidus (Group GP), lateral thalamus (Group LT), midbrain central gray (Group CG), substantia nigra (Group SN), pontine reticular formation (Group PRF), lateral pontomesencephalic area (Group LPMA), or medial longitudinal fasciculus (Group MLF). The ninth group (Group C) served as sham-operated controls, undergoing the same surgical procedure as the experimental groups, except for insertion of the lesion electrode and the application of current.

Postoperatively, the animals were housed, 2-3/cage, in medium-size wire cages containing a constant supply of food pellets and water. During the 1st postoperative week, a dish of sweetened wet mash was placed daily in each cage. Throughout the recovery period, the animals were handled for approximately $3 \mathrm{~min}$ on every 3rd day.

\section{Apparatus}

Photographs of the apparatus have been presented elsewhere (Thompson et al., 1984a). Briefly, the apparatus was divided into a startbox $(22.8 \times 23.0 \times 30.3 \mathrm{~cm})$, painted flat white, choice chamber $(61.6$ $\times 56.8 \times 30.3 \mathrm{~cm})$, painted flat white, and goalbox $(30.1 \times 56.8 \times$ $30.3 \mathrm{~cm}$ ), painted flat black. A clear Plexiglas guillotine door separated the startbox from the choice chamber. Interchangeable partitions in conjunction with a platform, cylinder, or ladder (each of which was painted flat white) could be positioned between the choice chamber and goalbox to form the three detour problems. During preliminary training, a partition containing a centrally located window $(10.4 \times 10.0 \mathrm{~cm})$ at floor level was employed. For Problem A, the partition used in preliminary training was placed in the apparatus along with a platform $(38.2$ $\times 58.8 \mathrm{~cm}$ ) that sloped upwards into the choice chamber to a maximum height of $10.1 \mathrm{~cm}$ above the floor. Problem B consisted of a partition containing a centrally located plastic cylinder $(4.6 \mathrm{~cm}$ in diam) that extended $20.3 \mathrm{~cm}$ into the choice chamber and was elevated $5.7 \mathrm{~cm}$ above the floor. Problem $\mathrm{C}$ consisted of a partition containing a window $(10.5 \times 7.7 \mathrm{~cm})$, located $10.3 \mathrm{~cm}$ above the floor, that could only be reached by climbing a vertically positioned ladder $(20.9 \times 7.7 \mathrm{~cm}$ with rungs spaced $2.0 \mathrm{~cm}$ apart) that extended $19.2 \mathrm{~cm}$ into the choice chamber. (For Problems B and C, a ramp located behind the partition allowed the rat to descend to the floor of the goalbox.) The entire apparatus was covered by a transparent Plexiglas lid and was illuminated by conventional ceiling fluorescent lights.

Activity was measured in a $56.0 \times 224.0 \mathrm{~cm}$ open field enclosed on three sides by the walls of the room and on the fourth side by a 32.5 $\mathrm{cm}$-high partition. The floor of the field was divided into $1628.0-\mathrm{cm}$ squares. Lighting was provided by conventional ceiling fluorescent lights.

\section{Procedure}

\section{Preliminary Training}

Following a 3-week recovery period, the animals were weighed and then deprived of water in their home cages for the duration of the learning experiment. After 2 days of deprivation, each animal was allowed to explore the entire apparatus (Day 1). A dish of water and a dish of sweetened wet mash were available in the goalbox from which the animal was permitted to ingest for $10 \mathrm{~min}$. On Day 2, the animals (usually run in squads of 2-3) were given 10 preliminary training trials with an in tertrial interval of 90-180 sec. At the beginning of each trial, the animal was inserted into the startbox and the startbox door was raised. In most instances, the animal would readily leave the startbox, traverse the choice chamber, enter the goalbox through the centrally located window, and ingest the water or mash. After $10 \mathrm{sec}$, the animal was carried to a restraining cage to await the next trial. On the 10th trial, the animals were usually allowed to ingest the water and mash for approximately $200 / \mathrm{sec}$ (This period, however, was shortened by at least $50 \%$ in those animals that were consistently reluctant to leave the startbox, slow in traversing the choice chamber, or hesitant in ingesting the water and mash during the course of preliminary training.)

\section{Detour Training}

Problem A was presented on Day 3, Problem B on Day 4, and Problem C on Day 5. Five trials were given on each problem with an intertrial interval of approximately $90-300 \mathrm{sec}$. The training procedure was the same as that described in preliminary training, except for the addition of the recording of response latencies-time between the raising of the startbox door and the animal's entrance into (all four legs placed beyond the threshold) the choice chamber.

With respect to Problem A, an error consisted of the animal's passing under the raised platform by at least the length of its body (excluding the tail). For Problem B, an error consisted of the animal's traversing beyond the opening of the elevated cylinder by at least the length of its body. An error on Problem C consisted of the animal's traversing beyond the ladder by at least the length of its body. Total (initial combined with repetitive) errors were recorded on each trial.

If an animal committed a total of 20 errors on Trial 1, 15 errors on Trial 2, 10 errors on Trial 3, or 5 errors on Trial 4 or Trial 5, it was assisted manually in mounting the platform (Problem A), entering the cylinder (Problem B), or climbing the ladder (Problem C). This was done in order to prevent the onset of "learned helplessness." However, the error score attained at the point of assistance was treated as though the animal had succeeded in negotiating the detour on that trial.

Finally, if an animal exhibited very little exploratory behavior on Trial 1 of Problem A (at least 5 min elapsing between the commission of one error and the next), it was discarded from the experiment. During training, the experimenter had no knowledge as to which group each subject belonged.

\section{Open-Field Test}

After completing training on Problem $C$, the animals were placed on ad-lib food and water. Twenty-four hours later (Day 6), each animal was placed in the center of the open field and allowed to explore during a 10-min period. The number of squares entered by all four paws was recorded. The number of rears (rising upon the hindlegs) was also counted.

\section{Swim Test}

After the activity test, each rat was checked for the integrity of labyrinthine righting reflexes by means of a "swim" test (see T'sang \& Wu, 1936). The rat was suspended by its tail and placed into a sink $(71.5 \mathrm{~cm}$ long $\times 40.7 \mathrm{~cm}$ wide) filled with water (at room temperature) to a depth of $20.0 \mathrm{~cm}$. If the subject floated or swam on top of the water (as normal rats usually do), a score of 0 was recorded. On the other hand, if the subject tucked its head downward while swimming on or beneath the surface (as rats with bilateral vestibular defects usually do), a score of 2 was recorded. A score of 1 was recorded when the subject dove toward the bottom without accompanying retroflexion of the head.

The swim test was given two times in succession, and a mean score was computed for each subject.

\section{Histology}

At the conclusion of postoperative testing, each brain-damaged rat was killed with an overdose of chloral hydrate, its vascular system perfused with normal saline followed by $10 \%$ Formalin, and the brain removed and stored in 10\% Formalin for 2-4 days. Each brain was then 
blocked, frozen, and sectioned frontally at $90 \mu$. Every fourth section through the lesioned area was retained and subsequently photographed at $12 \times$ by using the section as a negative film in an enlarger (see Thompson, 1971).

\section{RESULTS}

\section{Discarded Subjects}

Of the original 169 rats undergoing surgery, 39 died prior to the onset of the learning tests, the highest mortality rate occurring in Groups GP, SN, and PRF. Twentyfour animals were excluded from the experiment because their lesions were too small, grossly asymmetrical, or distant from the intended target area. Three additional animals were eliminated from the experiment because they showed little exploratory behavior (probably due to timidity) in the choice chamber on Trial 1 of Problem A: One came from Group C, one from Group LT, and one from Group GP.

\section{Body Weight}

After a 3-week recovery period, the mean body weight ( \pm standard error) of the controls was $146.2 \pm 4.6 \mathrm{~g}$ which, according to the Mann-Whitney U test, was significantly (ps $<.05$, two-tailed test) greater than that of Groups SN (112.1 $\pm 7.1 \mathrm{~g})$, MR $(103.0 \pm 5.8 \mathrm{~g})$, PRF $(110.2 \pm 6.1 \mathrm{~g}), \quad$ LPMA $(124.8 \pm 4.9 \mathrm{~g})$, and MLF $(117.8 \pm 5.2 \mathrm{~g})$. On the other hand, the body weights of Groups GP (138.7 $\pm 8.9 \mathrm{~g})$, LT $(158.8 \pm 5.8 \mathrm{~g})$, and CG $(134.6 \pm 5.9 \mathrm{~g})$ were not significantly different from that of the controls. (Unless specified otherwise, the MannWhitney two-tailed test was used for all comparisons made in this study.)

\section{Detour Performance}

\section{Error Scores}

Group C. With respect to Trial 1, the controls averaged fewer than 7 errors in solving each of the three detour problems (see Table 1). Only one animal required assistance (committing 20 errors) and that was on Problem B. As noted earlier (Thompson et al., 1984a),
Problem A tended to be the most difficult and Problem C the least difficult to solve, but the Wilcoxon test revealed that none of the differences in error scores approached statistical significance.

With respect to Trials 2-5, the controls averaged fewer than 2 errors on each problem. Fewer errors were committed on Problem C than on Problem A or Problem B $(\mathrm{p}<.05$, Wilcoxon test), but the difference in error scores associated with the latter two problems was not significant.

Group GP. Twelve rats sustained relatively discrete lesions to the globus pallidus, like the one shown in Figure 1. As noted in Table 1, these animals were significantly retarded in solving each of the three problems on Trial 1. They were also inferior to the controls on Trials 2-5. Six animals needed assistance on Problem B.

Group LT. The lateral dorsal and lateral posterior thalamic nuclei were extensively damaged in 11 rats. Figure 2 shows a representative lesion to this thalamic region. Performance on Problem A was not remarkably different from that of the controls. However, impaired performance was observed on Trials 2-5 of Problem B and on Trial 1 and Trials 2-5 of Problem C. Three animals needed assistance on Problem $B$ and two did on Problem C.

Group SN. Nine rats received relatively small lesions to the lateral half of the substantia nigra. In all cases, the underlying cerebral peduncle was slightly injured, such as the one shown in Figure 3. As a group, these animals performed poorly on Trial 1 and Trials $2-5$ on all three problems. Three needed assistance on Problem A, six on Problem B, and three on Problem C.

Group CG. The mesencephalic central gray was extensively damaged in 10 rats. In most cases, the overlying superior colliculus and adjacent reticular formation were only slightly injured (see Figure 4 for a representative lesion). Performance on Trial 1 of Problems A and $C$ was inferior to that of the controls. Except for Problem B, these animals were virtually indistinguishable from the controls on Trials 2-5. One animal needed assistance, and that was on Problem $A$.

Group PRF. Eight animals of this group sustained lesions to the pontine reticular formation similar to the one shown in Figure 5, while the remaining five had some-

Table 1

Mean Errors and Mean Response Latencies on Each Problem for All Groups

\begin{tabular}{|c|c|c|c|c|c|c|c|c|c|c|}
\hline \multirow[b]{3}{*}{ Group } & \multirow[b]{3}{*}{$\mathbf{N}$} & \multicolumn{3}{|c|}{ Problem A } & \multicolumn{3}{|c|}{ Problem B } & \multicolumn{3}{|c|}{ Problem C } \\
\hline & & \multicolumn{2}{|c|}{ Trial } & \multirow{2}{*}{$\begin{array}{c}\text { Response } \\
\text { Latency }\end{array}$} & \multicolumn{2}{|c|}{ Trial } & \multirow{2}{*}{$\begin{array}{c}\text { Response } \\
\text { Latency }\end{array}$} & \multicolumn{2}{|c|}{ Trial } & \multirow{2}{*}{$\begin{array}{c}\text { Response } \\
\text { Latency }\end{array}$} \\
\hline & & 1 & $2-5$ & & 1 & $2-5$ & & 1 & $2-5$ & \\
\hline C & 17 & $6.1 \pm 1.6$ & $1.6 \pm .4$ & $4.1 \pm 1.5$ & $5.5 \pm 1.2$ & $1.8 \pm .3$ & $3.8 \pm .8$ & $3.2 \pm .3$ & $.8 \pm .2$ & $2.3 \pm .1$ \\
\hline GP & 12 & $9.3 \pm 1.0^{*}$ & $4.2 \pm .5^{*}$ & $3.7 \pm .4$ & $13.1 \pm 2.0^{*}$ & $9.4 \pm 2.8^{*}$ & $4.7 \pm .7$ & $7.8 \pm 1.3^{*}$ & $3.4 \pm 1.0^{*}$ & $2.9 \pm .5$ \\
\hline LT & 11 & $7.0 \pm .8$ & $2.6 \pm .5$ & $4.4 \pm \quad, 9$ & $10.5 \pm 2.1$ & $5.5 \pm .8^{*}$ & $4.1 \pm .8$ & $8.4 \pm 1.7^{*}$ & $4.5 \pm 1.6^{*}$ & $3.1 \pm .7$ \\
\hline SN & 9 & $11.9 \pm 2.2^{*}$ & $3.7 \pm .5^{*}$ & $5.5 \pm .9$ & $17.7 \pm 1.2 *$ & $19.3 \pm 3.7^{*}$ & $5.4 \pm .8$ & $13.6 \pm 2.0^{*}$ & $7.7 \pm 2.5^{*}$ & $4.4 \pm .8$ \\
\hline CG & 10 & $10.3 \pm 1.4 *$ & $2.3 \pm .4$ & $1.8 \pm .1^{*}$ & $8.8 \pm 1.8$ & $3.4 \pm .5^{*}$ & $1.8 \pm .1$ & $6.1 \pm 1.2 *$ & $.6 \pm .1$ & $1.4 \pm .1$ \\
\hline PRF & 13 & $10.3 \pm 1.2^{*}$ & $4.2 \pm 1.2 *$ & $4.5 \pm .8$ & $8.6 \pm 1.0^{\prime}$ & $6.2 \pm 1.3^{*}$ & $3.7 \pm .7$ & $6.0 \pm 1.1 *$ & $3.0 \pm .9 *$ & $2.5 \pm .6$ \\
\hline MR & 11 & $14.6 \pm 1.9^{*}$ & $14.4 \pm 3.1^{*}$ & $3.9 \pm 1.2$ & $18.8 \pm .8$ & $26.4 \pm 3.2^{*}$ & $2.3 \pm .2$ & $17.0 \pm 1.4^{*}$ & $15.7 \pm 2.8^{*}$ & $2.0 \pm .4$ \\
\hline LPMA & 11 & $5.5 \pm 1.0$ & $1.4 \pm .2$ & $2.5 \pm .4$ & $6.4 \pm 1.8$ & $2.9 \pm 1.0$ & $2.3 \pm .3$ & $5.6 \pm 1.2$ & $1.1 \pm .4$ & $1.5 \pm .2$ \\
\hline MLF & 9 & $6.7 \pm 1.3$ & $2.7 \pm .5$ & $1.8 \pm .2^{*}$ & $9.0 \pm 1.1^{*}$ & $5.3 \pm .7 *$ & $1.7 \pm .2$ & $8.9 \pm 2.0^{*}$ & $3.1 \pm .7^{*}$ & $1.4 \pm .1$ \\
\hline
\end{tabular}

${ }^{*}$ Differed from the controls at least at the .05 level. 


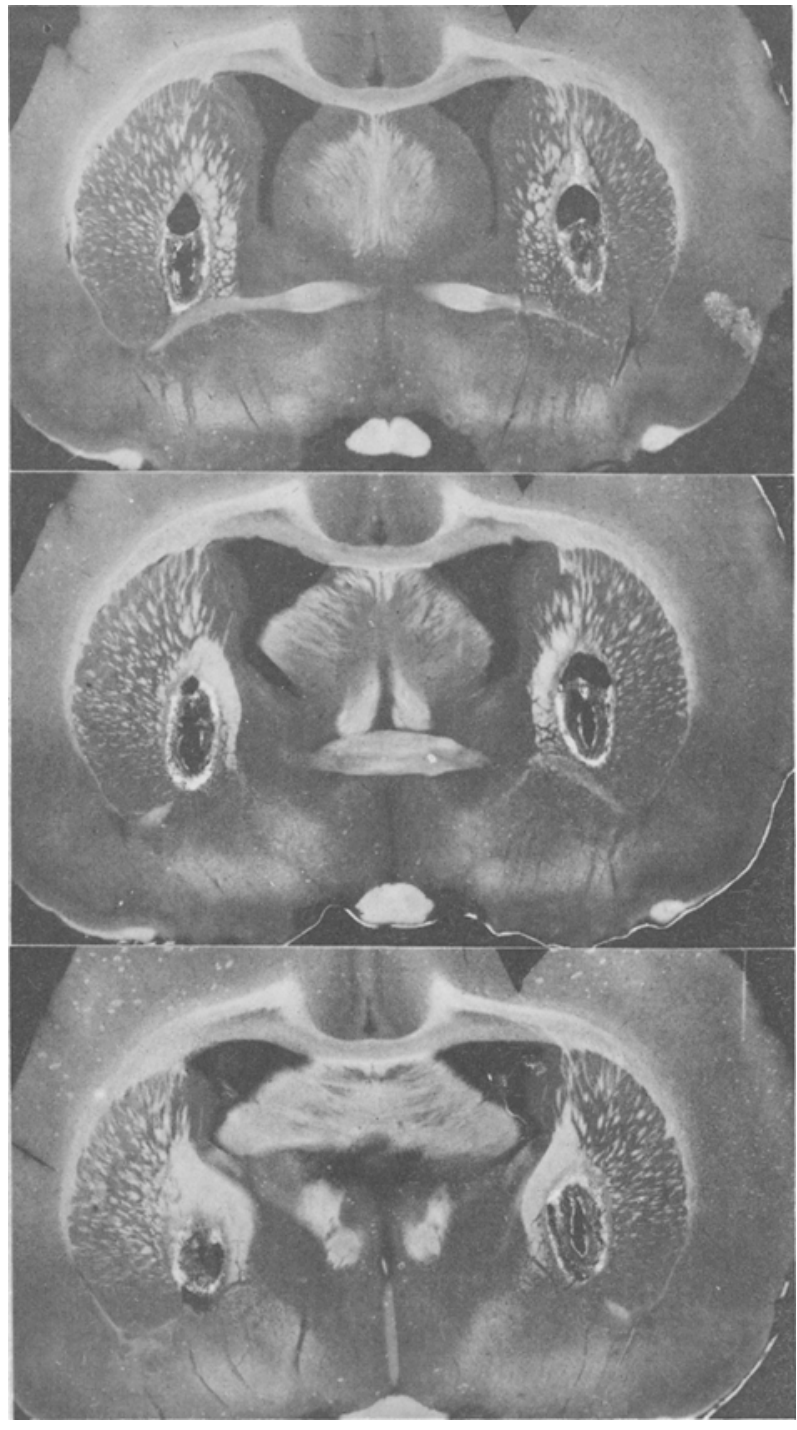

Figure 1. Photographs of unstained sections showing a lesion to the globus pallidus in one rat.

what more anteriorly and ventrally placed lesions which began at the level of the caudal half of the interpeduncular nucleus. (There was a suggestion that the posterior lesions tended to produce greater performance deficits than the anterior lesions, but none of the differences in error scores reached statistical significance.) As a group, these animals were deficient in solving all detour problems on Trial 1 as well as on Trials 2-5. Only one animal needed assistance, and that was on Problem A.

Group MR. All 11 animals suffered varying amounts of damage to the median raphe. In six cases, such as the one shown in Figure 6, the lesions began at the rostral portion of the median raphe and extended caudally to the level of the dorsal tegmental nucleus of Gudden. In the remaining cases, the lesions began dorsal to the interpeduncular nucleus and extended caudally into the me-

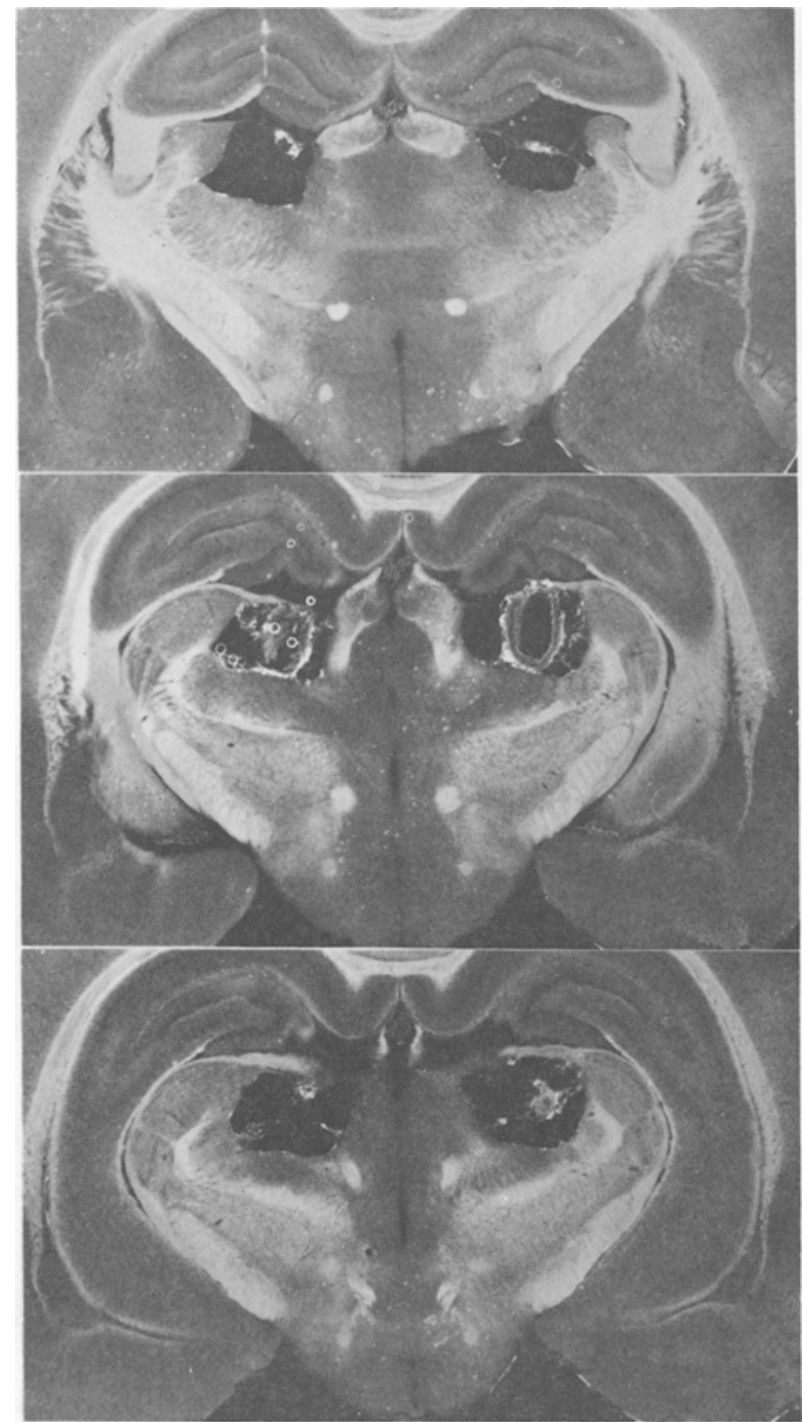

Figure 2. Photographs of unstained sections showing a lesion to the lateral thalamus in one rat.

dian raphe. (There was no indication that the posterior lesions produced greater performance deficits than the anterior lesions.) This group was profoundly impaired in solving each of the three detour problems on Trial 1 as well as on Trials 2-5. Six animals needed assistance on Problem A, nine on Problem B, and seven on Problem C.

As shown in Figure 7, the learning curves of Group MR were, on the whole, markedly separated from those of the previously discussed groups with damage to components of the general learning system.

Group LPMA. All 11 animals of this group suffered massive lesions to the far-lateral pontomesencephalic region like the one shown in Figure 8. These animals performed as well as the controls on all three problems. One animal needed assistance, and that was on Problem B.

Group MLF. The medial longitudinal fasciculus was 


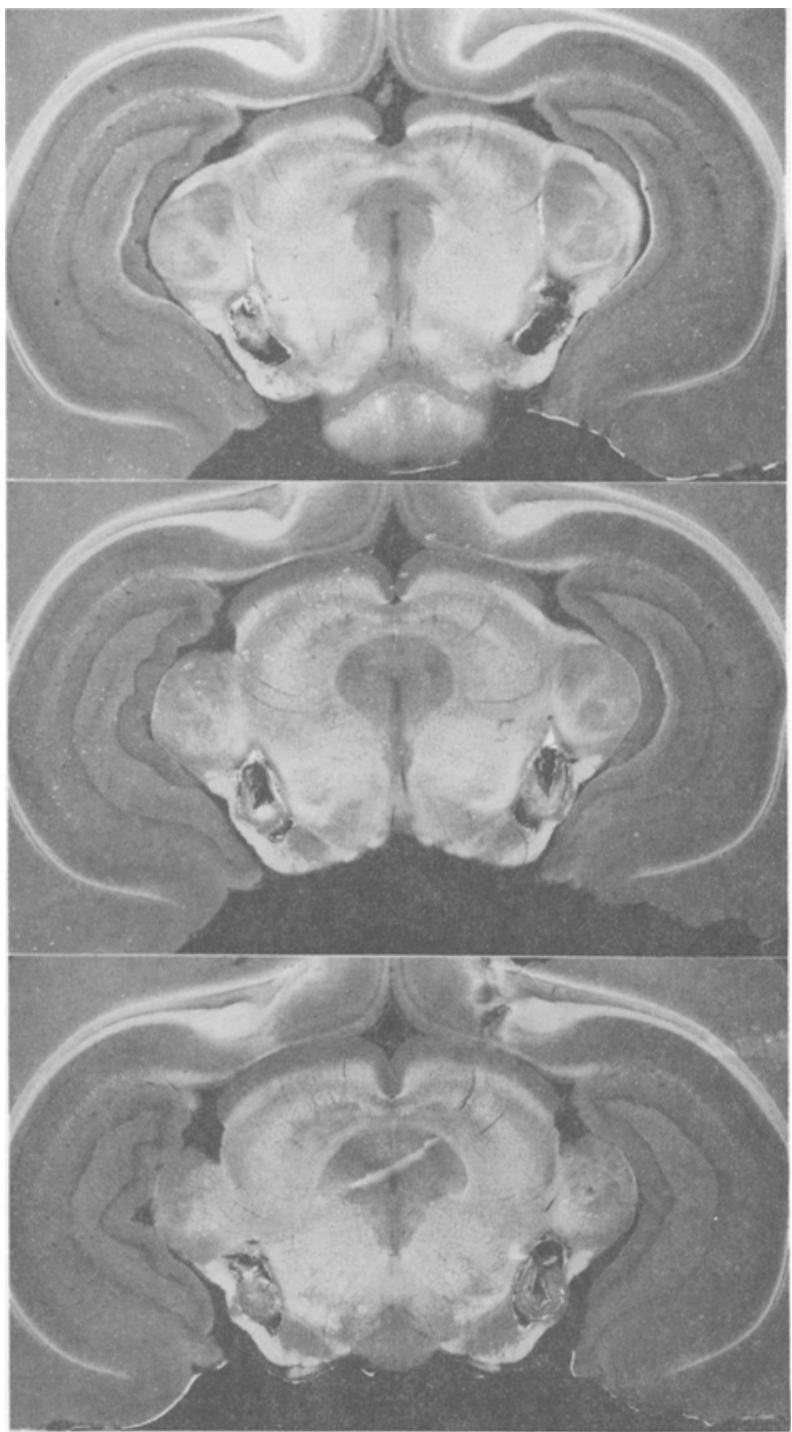

Figure 3. Photographs of unstained sections showing a lesion to the substantia nigra in one rat.

bilaterally damaged in all nine animals (see Figure 9 for a representative lesion). Although performing as well as the controls on Problem A, this group was inferior to the controls on Trial 1 and Trials 2-5 of Problems B and C. One animal needed assistance, and that was on Problem C.

\section{Latency Scores}

Time elapsing between elevation of the startbox door and entrance into the choice chamber was recorded on each trial for the purpose of providing some objective measure of motivation. As noted in Table 1, only Group SN exhibited significantly longer latency scores than the controls, but this appeared only on Problem C. Interestingly, Groups CG and MLF showed significantly shorter latency scores than the controls on Problem A.

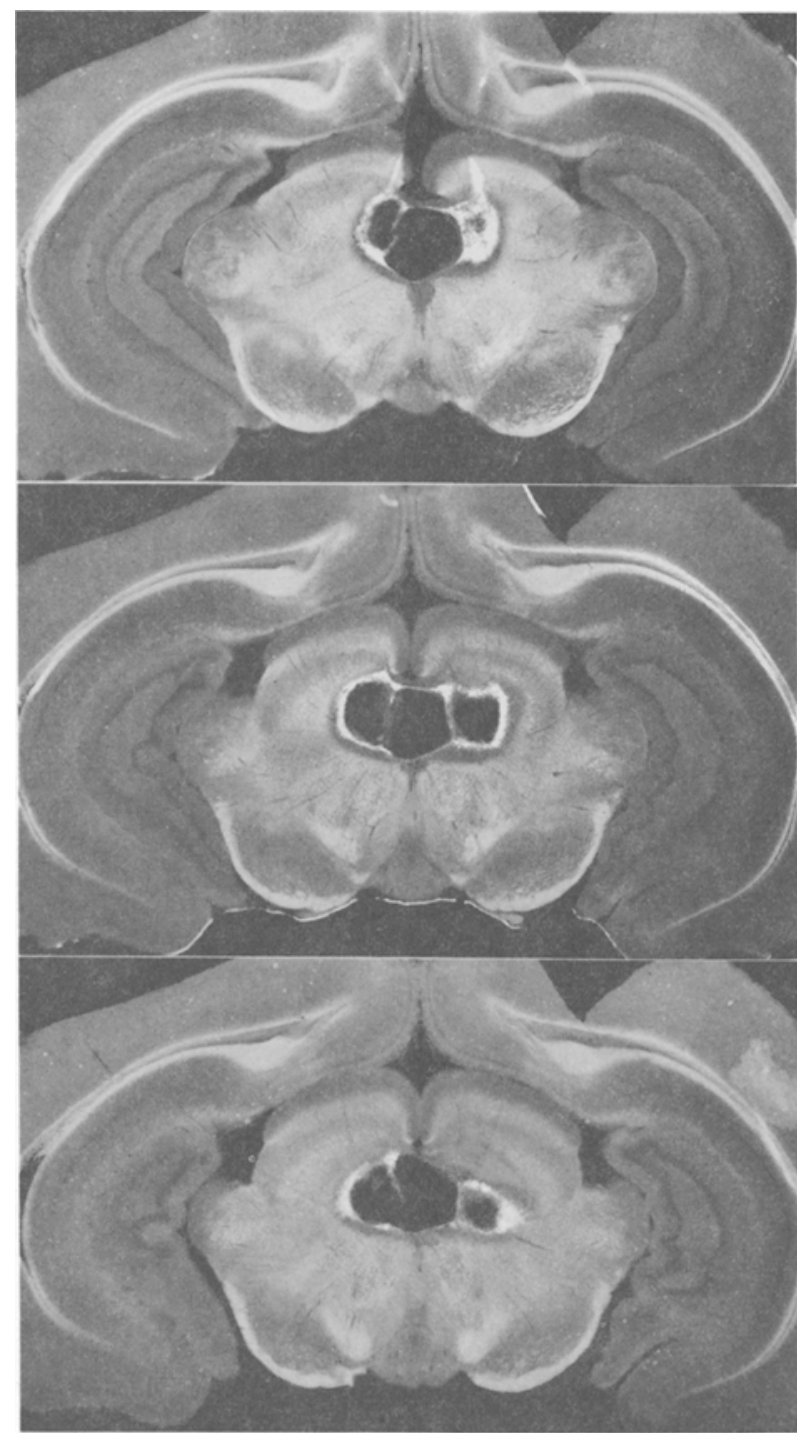

Figure 4. Photographs of unstained sections showing a lesion to the midbrain central gray in one rat.

\section{Behavior in the Choice Chamber}

We have previously described in some detail our impressions of the behavior of those neocortically damaged rats that were retarded in solving the detour problems on Trial 1 (Thompson et al., 1984a). That description would also apply to the behavior of those brainstem-damaged rats of the present study that were deficient in solving the detour problems on Trial 1. First of all, these brainstemdamaged rats tended to commit more errors than the controls before making initial contact (sniffing, touching with forepaws, or mounting) with the significant parts of the problem situation (leading edge of the platform on Problem A, rim of the cylinder on Problem B, and rungs of the ladder on Problem C). Secondly, these rats tended to be less persistent than the controls in responding to these significant parts - their reactions were momentary dur- 


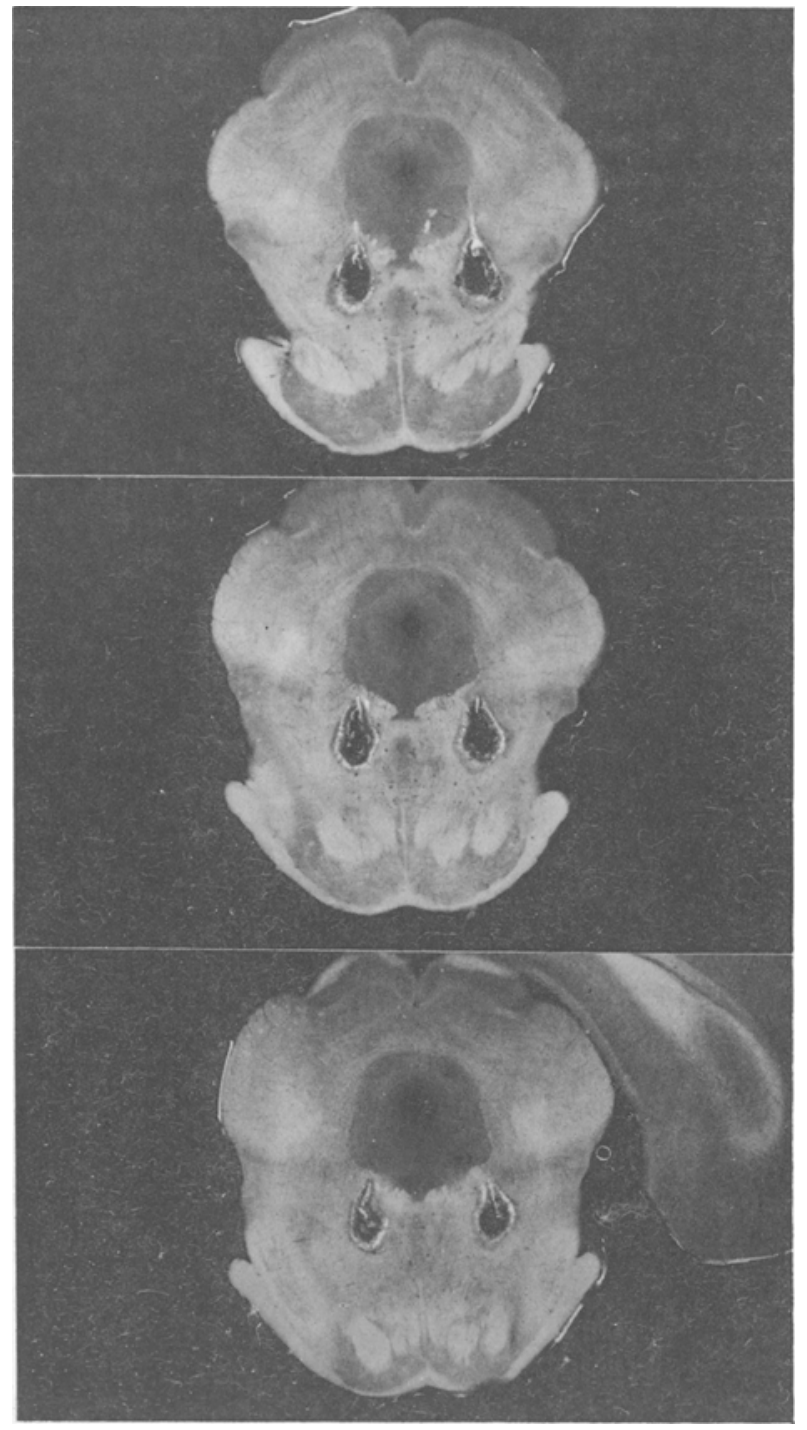

Figure 5. Photographs of unstained sections showing a lesiion to the pontine reticular formation in one rat.

ing the first few contacts. (It should be emphasized that these observations were not based upon objective records and therefore should be interpreted with caution.)

\section{Activity and Rears}

As shown in Table 2, none of the brain-damaged groups was significantly less active than the controls in the openfield test. (The relatively low scores of Groups MR and MLF were due mainly to the performance of three animals from each group that entered fewer than 60 squares in the 10-min period.) Interestingly, Groups GP and CG were significantly more active than the controls.

On the other hand, Groups MR, PRF, and LPMA made significantly fewer rearing responses than the controls in the open-field test. Only those animals with lateral tha-

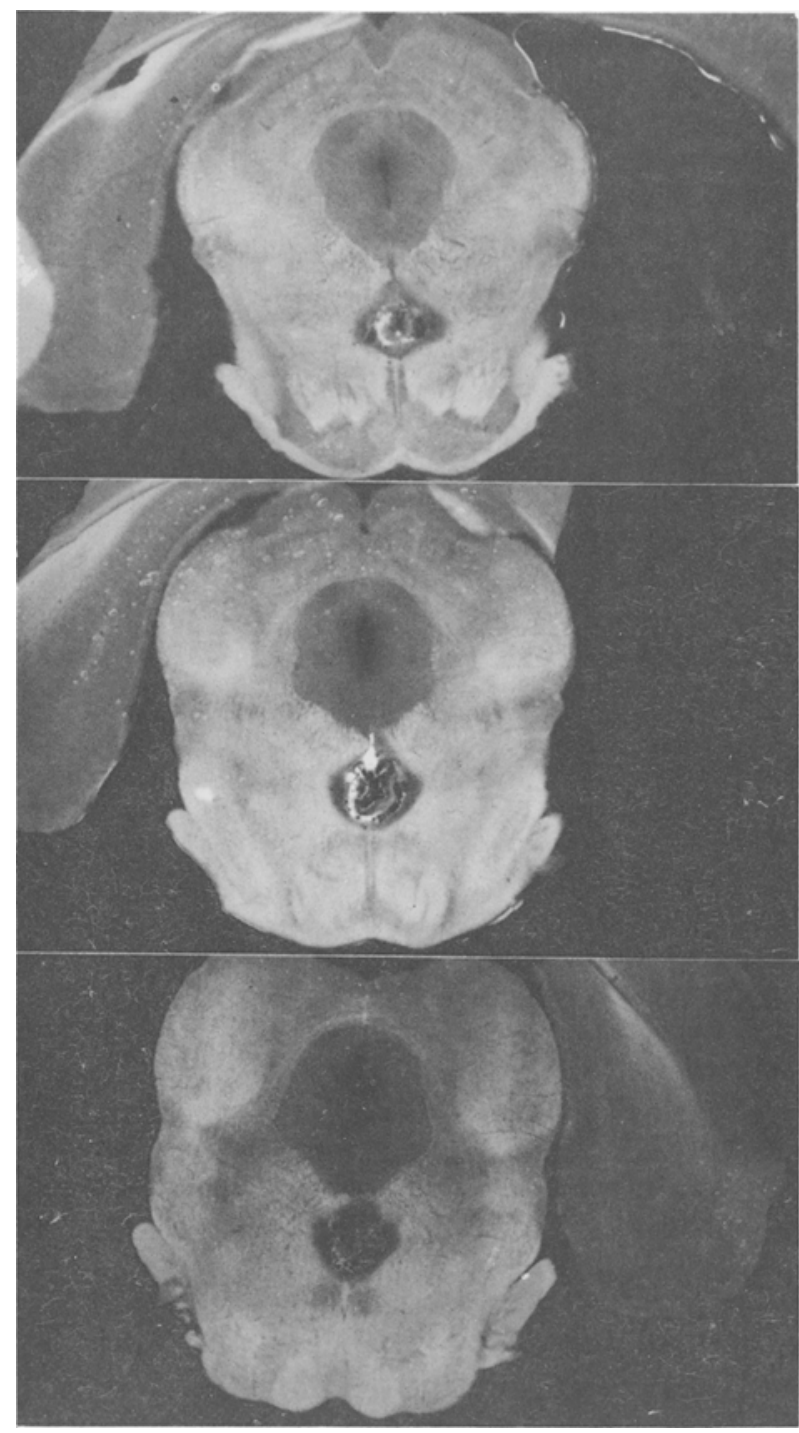

Figure 6. Photographs of unstained sections showing a lesion to the median raphe in one rat.

lamic lesions made significantly more rearing responses than the controls.

Table 2

Mean Open Field Activity and Swim Test Scores for All Groups

\begin{tabular}{lcrrrrrr} 
Group & $\begin{array}{c}\text { Squares } \\
\text { Entered }\end{array}$ & Range & Rears & Range & $\begin{array}{c}\text { Swim } \\
\text { Test }\end{array}$ & Range \\
\hline C & 146.4 & $6-330$ & 25.5 & $1-49$ & .1 & $0-1$ \\
GP & $173.5^{*}$ & $96-257$ & 26.1 & $8-45$ & .1 & $0-1$ \\
LT & 150.8 & $12-246$ & $34.6^{*}$ & $6-58$ & .2 & $0-1$ \\
SN & 158.4 & $95-308$ & 21.8 & $17-29$ & .5 & $0-2$ \\
CG & $257.4^{*}$ & $76-342$ & 29.7 & $21-45$ & .1 & $0-1$ \\
PRF & 135.0 & $32-307$ & $11.8^{*}$ & $2-32$ & $1.9^{*}$ & $1-2$ \\
MR & 117.6 & $36-257$ & $7.5^{*}$ & $3-20$ & $1.4^{*}$ & $1-2$ \\
LPMA & 148.1 & $33-262$ & $10.2^{*}$ & $4-31$ & .2 & $0-1$ \\
MLF & 115.6 & $12-231$ & 17.1 & $6-46$ & $1.8^{*}$ & $1-2$ \\
\hline
\end{tabular}

*Differed from the controls at least at the.05 level. 


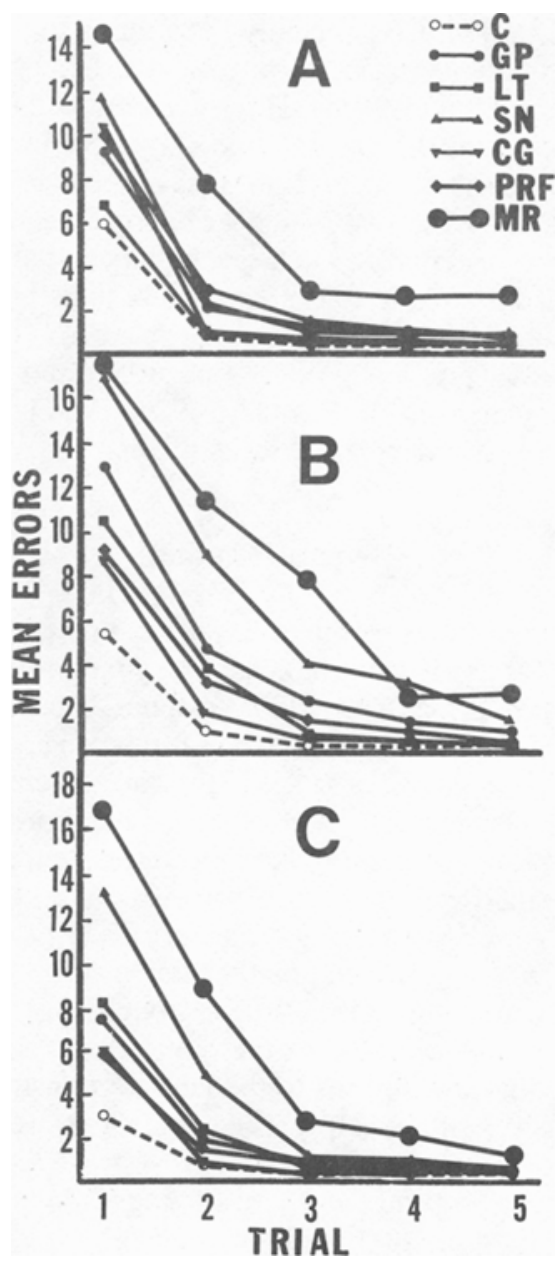

Figure 7. Mean errors on Problems A, B, and C for the controls and those groups receiving damage to the general learning system.

\section{Swim Test}

As expected from an earlier report (Thompson et al., 1976), all animals from Groups MR, PRF, and MLF showed a defective labyrinthine righting reflex during the swim test (see Table 2).

\section{DISCUSSION}

\section{Preliminary Considerations}

It is important to note at the outset that the solution of our detour problems on Trial 1 appears to require a cognitive function that is qualitatively different from that required on Trials 2-5. In the former case, efficiency in finding the correct pathway to the goalbox is dependent upon the readiness of the animal to abandon unsuccessful responses and to try new ones until the correct one is found. This ability, which has been aptly termed "response flexibility" (Riopelle, 1967, p. 253), proba- bly involves such psychological processes as attention, behavioral inhibition, exploration, and working memory (see Thompson et al., 1984a). In contrast, efficiency in finding the correct pathway on Trials 2-5 would depend largely upon the extent of "associative learning"' (reference memory) that took place during the time that the animal was making the correct and incorrect responses on the preceding trial(s). Evidence supporting such a distinction comes from the findings that neocortically damaged rats, while tending to show a marked deficit in solving the climbing detour problems on Trial 1 , perform about as well as the controls on Trials 2-5 (Thompson et al., 1984a).

It is also noteworthy to mention that visual cues seem to be relevant mainly for the solution of Problem B. This

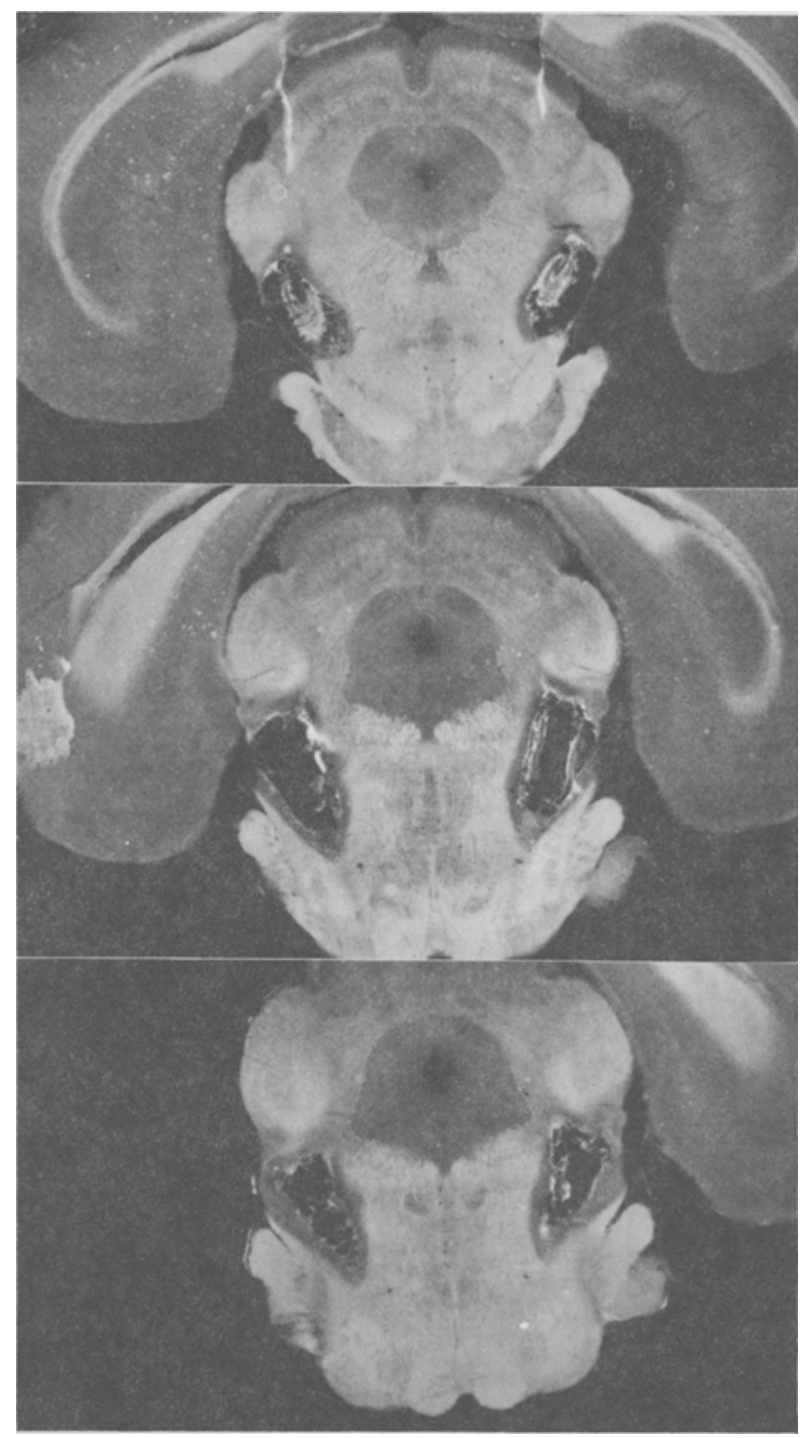

Figure 8. Photographs of unstained sections showing a lesion to the lateral pontomesencephalic area in one rat. 


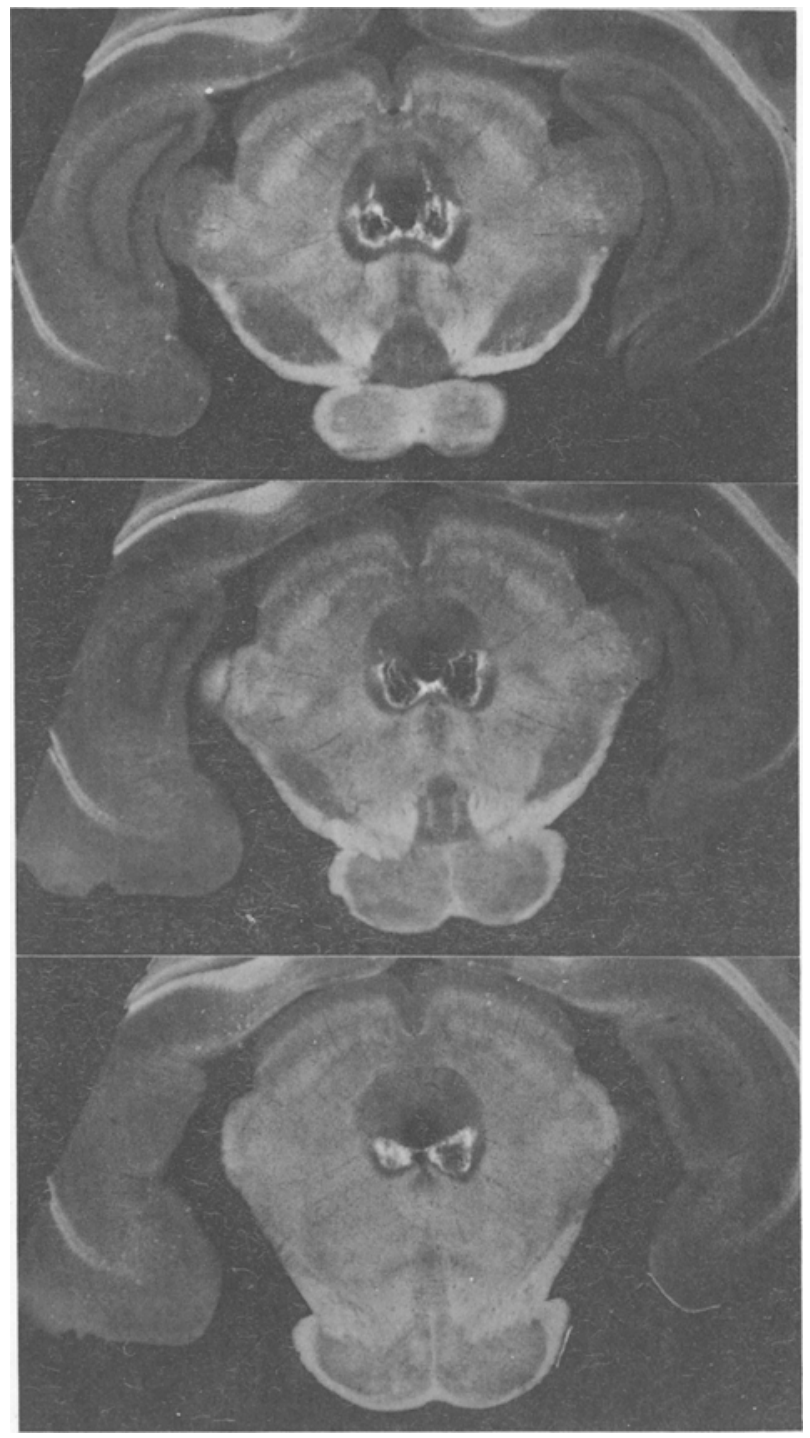

Figure 9. Photographs of unstained sections showing a lesion to the medial longitudinal fasciculus in one rat.

conclusion is based on the observations that weanling rats subjected to either enucleation of the eyes (Thompson, Harmon, \& Yu, 1984b) or bilateral aspiration of the occipital cortex (Thompson et al, 1984a) are significantly impaired in finding the correct route (on Trial 1 and Trials 2-5) on Problem B, are unimpaired on Problem A, and show only a mild impairment on Trials 2-5 of Problem C.

It can be argued that our detour problems are similar to simple spatial mazes, since the former contain a true path and a "blind alley." These problems can also be viewed as variants of spatial reversal tasks to the extent that the pretrained response of running to the centrally located window must be suppressed and a new response to an alternate pathway must be learned. Some evidence supporting these similarities comes from the findings that hippocampal lesions, which are known to impair acquisition of maze habits and spatial reversal tasks (O'Keefe
\& Nadel, 1978), have recently been reported to produce profound deficits in the learning of our detour problems (Thompson et al., 1984a). However, it would be premature to conclude that the solution of our detour problems requires the same cognitive abilities as those required for the solution of maze habits and spatial reversal tasks. This is shown by the findings that rats with occipital ablations or lateral thalamic lesions are virtually indistinguishable from the controls in learning Problem A (Thompson et al., 1984a; present study), but are significantly impaired in learning maze habits (Lashley, 1929; Thompson \& Yu, 1983) and spatial reversal tasks (Thompson, 1983a).

\section{General Findings}

In light of the foregoing considerations, the results of the current experiment indicate that young rats with circumscribed lesions to the globus pallidus, substantia nigra, median raphe, or pontine reticular formation are markedly deficient in both response flexibility and associative learning. This conclusion is based on the observations that those groups sustaining damage to these brainstem sites committed significantly more errors than the controls on Trial 1 as well as on Trials $2-5$ before discovering the correct pathway on each of the three detour problems. Nonspecific debilitating effects arising from brainstem damage cannot readily account for these observations, since the group with large lesions to the lateral brainstem at pontomesencephalic levels earned excellent performance scores on all three problems.

It is also doubtful that these findings can be explained in terms of a motor disturbance because (1) none of our brainstem-damaged rats exhibited a locomotor deficit involving the forelimbs or hindlimbs, either during the detour tests or during the open-field test, (2) on Trials 25 , most of our brainstem-damaged rats were virtually indistinguishable from the controls in their ability to perform the particular climbing response required on each problem, and (3) the presence of a motor impairment would lead to the expectation that performance on Trial 1 of Problem A would be inferior to that on Trial 1 of Problem B (the vertical distance between supporting surfaces on Problem $A$ is $4.4 \mathrm{~cm}$ greater than that on Problem B); yet Groups GP, SN, and MR tended to make more errors on Problem B than on Problem A.

Similarly, no conspicuous relationship was found between detour problem-solving ability and such variables as activity, motivation, body weight, rearing responses, or labyrinthine righting reflexes. In the case of activity, none of the brain-damaged groups was found to be hypoactive relative to the controls in the open-field test and only Groups GP and CG evidenced hyperactivity. In terms of latency scores recorded on Problems A and B, all brain-damaged groups appeared to be at least as motivated as the controls to enter the choice chamber. With respect to body weight, Groups SN, MR, PRF, LPMA, and MLF were significantly smaller than the controls, but the latter two groups performed about as well as the con- 
trols on Problem A. Rearing responses were significantly reduced in frequency in Groups MR, PRF, and LPMA, but again, the latter group earned excellent performance scores on all three problems. And, finally, the fact that Group MLF performed as well as the controls on Problem A despite the presence of a defective labyrinthine righting reflex suggests that such a defect cannot entirely account for the poor performance scores earned by Groups MR and PRF.

As noted in Figure 7, those rats with lesions to the substantia nigra or median raphe showed the most profound deficits in detour problem-solving behavior. Each of these groups made significantly more pooled errors on Trials 15 than any other brain-damaged group studied in this experiment (ps < .05). Furthermore, while none of the rats in Groups C, GP, LT, CG, PRF, LPMA, or MLF needed assistance on Trial 1 on at least two problems, 4 of the 9 animals in Group SN and 8 of the 11 animals in Group MR did; these differences in proportions existing between Group SN (or Group MR) and each of the remaining groups are significant at least at the .05 level (Fisher exact probability test). A comparison of Group SN with Group MR revealed that the latter made significantly more pooled errors on Trials 2-5 than the former ( $p<$ .01 ), but the difference in pooled errors committed on Trial 1 fell considerably short of statistical significance.

There is increasing evidence that lesions to the median raphe produce a pattern of behavioral deficits on an assortment of laboratory tasks similar to that produced by hippocampal lesions (see Wirtshafter \& Asin, 1983). The results of the current study add to this pattern to the extent that median raphe lesions were found to induce impairments in detour problem-solving behavior as striking as those induced by hippocampal lesions (Thompson et al., 1984a). However, a visual discrimination task appears to differentiate rats with large median raphe lesions from rats with large dorsal or ventral hippocampal lesions: The former exhibit dramatic deficits in acquisition (Thompson, 1982b) and retention (Thompson, 1978) of a white-black discrimination, whereas the latter do not exhibit these deficits (Thompson, 1978, 1982a, 1982b). Conceivably, damage to certain elements within the median raphe (serotonergic cells, nonserotonergic cells, or fibers in passage) may be responsible for the pattern of deficits similar to that associated with hippocampal lesions, whereas damage to other elements may be responsible for the visual discrimination impairments. Alternatively, it is possible that destruction of certain (or all) elements of the median raphe disorganizes a unitary function that contributes to the normal acquisition and retention of a broad class of laboratory tasks, of which visual discriminations and detour problems are a part.

\section{The General Learning System}

Earlier studies have demonstrated that lesions to the globus pallidus, lateral thalamus, substantia nigra, midbrain central gray, median raphe, or pontine reticular forma- tion in weanling (Thompson et al., 1984; Thompson \& $\mathrm{Yu}, 1983$ ) or adult (Thompson, 1982a, 1982b; Thompson et al., 1983) rats produce learning impairments on visual as well as nonvisual discrimination habits. These results have provided the basis for the hypothesis that the foregoing structures play a nonspecific role in the learning process and that they constitute the major portion of the general learning system (GLS) of the rat brain. Supporting evidence has come from the findings that damage to any one of these structures in weanling rats retards acquisition of a 3-cul maze habit (Thompson et al., 1984; Thompson \& Yu, 1983). Further support for the inclusion of the globus pallidus, substantia nigra, median raphe, and pontine reticular formation within the rodent's GLS has emerged from the findings of the current experiment which showed that selective lesions to these structures led to significant deficits on Trial 1 and Trials 2-5 on Problems A, B, and C. On the other hand, damage to the lateral thalamus or midbrain central gray yielded more variable effects. The animals of Group LT showed no deficit on Problem A, nor did they perform significantly worse than the controls on Trial 1 of Problem B. Group CG failed to show a significant deficit on Trial 1 of Problem B and performed as well as the controls on Trials 2-5 of Problems A and C. Since the lesions suffered by Groups LT and CG were comparable in both topography and magnitude to those investigated in our earlier study on weanling rats (Thompson \& Yu, 1983), these erratic effects on detour problem-solving behavior question the importance of the lateral thalamus and midbrain central gray in the overall learning process, and justify the removal of these structures from the GLS.

The foregoing findings, which implicate the globus pallidus, substantia nigra, median raphe, and pontine reticular formation in the general learning process, are significant for a number of reasons. First, it should be recognized that a generalized learning impairment induced by specific brain lesions is not a common occurrence: Rats with discrete lesions to the cerebral cortex, limbic forebrain, caudoputamen, nucleus accumbens, thalamus, medial hypothalamus, dorsal midbrain, lateral pontomesencephalic area, or cerebellar cortex have not been found to exhibit a generalized learning impairment (Thompson, 1982a, 1982b; Thompson et al., 1983; current study). Such a localized effect suggests that the neuronal elements intrinsic to (or extraneous fibers passing through) the regions of the globus pallidus, substantia nigra, median raphe, and pontine reticular formation are potentially more involved in the ability to learn diverse laboratory tasks than those associated with most other regions of the brain.

Second, lesions to the globus pallidus, substantia nigra, median raphe, or pontine reticular formation produce not only a generalized learning impairment, but a generalized retention impairment as well (Thompson, 1978, 1983b). These results suggest that the learning impairments arising from lesions to the foregoing structures may be referable to interference not only with those central 
processes concerned with short-term (working) memory, but with those processes concerned with storage or retrieval of learning experiences as well.

Third, if it is granted that a common pattern of acquisition deficits arising from focal lesions to different brain sites expose functional interrelationships among the brain sites involved, then the globus pallidus, substantia nigra, median raphe, and pontine reticular formation may constitute a neural system that performs a particular (though undoubtedly complex) function essential for the general learning process. One of us (Thompson, 1984) has already argued that the functional significance of the GLS is related to the mediation of anticipatory sets as conceived by Sperry (1955).

Fourth, the presence of a generalized learning and retention loss attending damage to the globus pallidus, substantia nigra, median raphe, or pontine reticular formation in the albino rat may have a bearing on the current controversy concerning whether dementia is strictly a reflection of the degeneration of cortical neurons or whether it can also be the outcome of subcortical neuropathology (Benson, 1983). The finding that progressive mental deterioration occurs in numerous cases in which the major lesions lie at the subcortical level, as in Parkinsonism (Benson, 1984), progressive supranuclear palsy (Steele, Richardson, \& Olszewski, 1964), and Hallervorden-Spatz syndrome (Dooling, Schoene, \& Richardson, 1974), has led to the concept of a "subcortical dementia" (Albert, 1978). Among the subcortical structures involved are the globus pallidus, substantia nigra, and reticular formation of the brainstem. However, since cortical neurons may also show abnormal features in these clinical cases (Mayeux, Stern, Rosen, \& Benson, 1983), the notion that the pallidal, nigral, and reticular areas participate in cognitive functions has not been generally accepted. Although our data on this issue are far from being compelling, they suggest at the very least that subcortical encephalopathy cannot be excluded as a possible cause of intellectual dysfunction in dementia.

Finally, the finding that damage to the globus pallidus, substantia nigra, median raphe, or pontine reticular formation in weanling rats leads to learning impairments on a wide range of laboratory tasks poses the possibility that the weanling rat bearing these lesions may serve as an animal model for certain classes of mental retardation. From a pathogenic point of view, this model would seem to be less than satisfactory to the extent that mental retardation has not been linked to neuropathology of subcortical formations (see, however, Buchwald, 1975). On the other hand, the model does have some merit from a behavioral perspective, since the mental retardate and the brain-injured rat (one with lesions to the GLS) evidence a generalized learning impairment. A further similarity could come from the discovery that the weanling rat with GLS lesions suffers from a deficit in both inhibitory and attentional processes. (Preliminary studies in our laboratory suggest that extinction of a runway habit as well as performance on a series of visual discriminations of in- creasing difficulty are impaired in weanling rats with lesions to the globus pallidus, substantia nigra, median raphe, or pontine reticular formation.) This finding would be of considerable interest since mental retardates have been hypothesized to exhibit deficits in behavioral inhibition (Denny, 1964) and attention (Zeaman \& House, 1979).

\section{REFERENCES}

AlberT, M. L. (1978). Subcortical dementia. In R. Katzman, R. D. Terry, \& K. L. Bick (Eds.), Alzheimer's disease: Senile dementia and related disorders (pp. 173-180). New York: Raven Press.

Benson, D. F. (1983). Subcortical dementia: A clinical approach. $A d-$ vances in Neurology, 38, 185-194.

Benson, D. F. (1984). Parkinsonian dementia: Cortical or subcortical? Advances in Neurology, 40, 235-240.

BUCHWALD, J. S. (1975). Brainstem substrates of sensory information processing and adaptive behavior. In N. A. Buchwald \& M. A. B Brazier (Eds.), Brain mechanisms in mental retardation (pp. 315-333) New York: Academic Press.

DENNY, M. R. (1964). Research in learning and performance. In $\mathbf{H}$ H. Stevens \& R. Heber (Eds.), Mental retardation (pp. 100-142). Chicago: University of Chicago Press.

Dooling, E. C., Schoene, W. C., \& Richardson, E. P. (1974) Hallervorden-Spatz syndrome. Archives of Neurology, 30, 70-83.

LASHLEY, K. S. (1929). Brain mechanisms and intelligence. Chicago University of Chicago Press.

Mayeux, R., Stern, Y., Rosen, J., \& Benson, D. F. (1983). Is “subcortical dementia" a recognizable clinical entity? Annals of Neurology, 14, 278-283.

O'KEEFE, J., \& NADEL, L. (1978). The hippocampus as a cognitive map. Oxford: Clarendon Press.

RIOPELle, A. J. (1967). Animal problem solving. Baltimore: Penguin SPERRY, R. W. (1955). On the neural basis of the conditioned response British Journal of Animal Behaviour, 3, 41-44.

Steele, J. C., Richardson, J. C., \& Olszewski, J. (1964). Progressive supranuclear palsy. Archives of Neurology, 10, 333-359.

THOMPson, R. (1971). Introducing subcortical lesions by electrolytic methods. In R. D. Myers (Ed.), Methods in psychobiology (Vol. 1 pp. 131-154). New York: Academic Press.

THOMPSON, R. (1978). A behavioral atlas of the rat brain. New York: Oxford University Press.

Thompson, R. (1982a). Brain lesions impairing visual and spatial reversal learning in rats: Components of the "general learning system" of the rodent brain. Physiological Psychology, 10, 186-198.

Thompson, R. (1982b). Impaired visual and spatial reversal learning in brain-damaged rats: Additional components of the "general learning system" of the rodent brain. Physiological Psychology, 10, 293-305.

Thompson, R. (1983a). Abnormal learning and forgetting of individual spatial reversal problems in brain-damaged rats. Physiological Psychology, 11, 35-46.

Thompson, R. (1983b). Brain systems and long-term memory. Behavioral and Neural Biology, 37, 1-45.

THOMPson, R. (1984). Nonspecific neural mechanisms involved in learning and memory in the rat. In N. Butters \& L. Squire (Eds.), Neu ropsychology of memory (pp. 408-416). New York- Guilford Press.

Thompson, R., ARABIE, G. J., \& SiSK, G. B. (1976). Localization of the "incline plane discrimination memory system"' in the white rat. Physiological Psychology, 4, 311-324.

Thompson, R., Gallardo, K., \& Yu, J. (1983). Posterolateral hypothalamic and midbrain central gray lesions impair visual and spatial reversal learning: Further additions to the "general learning system" of the rodent brain. Physiological Psychology, 93-102.

Thompson, R., HaRMon, D., \& YU, J. (1984a). Detour problem-solving behavior in rats with neocortical and hippocampal lesions: A study of response flexibility. Physiological Psychology, 12, 116-124. 
THOMPSON, R., HARMON, D., \& YU, J. (1984b). The role of vision in detour problem-solving behavior. Unpublished manuscript.

THOMPSON, R., RAMSAY, A., \& YU, J. (1984). A generalized learning deficit in albino rats with early median raphe or pontine reticular formation lesions. Physiology \& Behavior, 32, 107-114.

THOMPSON, R., \& YU, J. (1983). Specific brain lesions producing nonspecific (generalized) learning impairments in weanling rats. Physiological Psychology, 11, 225-234.

T'sang, Y., \& WU, C. (1936). The effects of unilateral labyrinthectomy in the albino rat. Chinese Journal of Physiology, 10, 571-598.
WirTShafTer, D., \& Asin, K. E. (1983). Impaired radial maze performance in rats with electrolytic median raphe lesions. Experimental Neurology, 79, 412-421.

Zeaman, D., \& House, B. J. (1979). A review of attention theory. In N. R. Ellis (Ed.), Handbook of mental deficiency (2nd ed., pp. 63120). Hillsdale, NJ: Erlbaum.

(Manuscript received June 25, 1984;

revision accepted for publication September 5, 1984.) 